\title{
Transition regimes for growing crack populations
}

\author{
Chrysanthe Spyropoulos* \\ Department of Applied Physics and Applied Mathematics, Columbia University, New York, New York 10027 \\ Christopher H. Scholz ${ }^{\dagger}$ and Bruce E. Shaw ${ }^{\dagger}$ \\ Lamont-Doherty Earth Observatory, Columbia University, Palisades, New York 10964
}

(Received 10 October 2001; published 3 May 2002)

\begin{abstract}
Numerous observational papers on crack populations in the material and geological sciences suggest that cracks evolve in such a way as to organize in specific patterns. However, very little is known about how and why the self-organization comes about. We use a model of tensilelike cracks with friction in order to study the time and space evolution of normal faults. The premise of this spring-block analog is that one could model crustal deformation for long time scales assuming a brittle layer coupled to a ductile substrate. The long time-scale physics incorporated into the model are slip-weakening friction, strain-hardening rheology for coupling the two layers, and randomly distributed yield strength of the brittle layer. We investigate how the evolution of populations of cracks depends on these three effects, using linear stability analysis to calculate the stable regimes for the friction as well as numerical simulations to model the nonlinear interactions of the cracks. We find that we can scale the problem to reduce the relevant parameters to a single one, the slip weakening. We show that the distribution of lengths of active cracks makes a transition from an exponential at very low strains, where crack nucleation prevails, to a power law at low to intermediate strains, where crack growth prevails, to an exponential distribution of the largest cracks at higher strains, where coalescence dominates. There is evidence of these different length distributions in continental and oceanic normal faults. For continental deformation the strain is low, and the faults have power-law frequency-size distributions. For mid-ocean ridge flanks the strain is greater, up to an order of magnitude higher than the continental strain, and faults have exponential-like frequency-size distributions. No theory has been offered to explain this difference in the distributions of continental and mid-ocean faults. In this paper we argue that they are indicative of different stages of evolution. The former faults are at an early stage of relatively small deformation, while the latter are at a later stage of the evolution. For high strain the faults reach a saturation regime with system size cracks evenly spaced in proportion to the brittle layer thickness. We asymptotically approximate the time space evolution of faults as a long time-scale phenomenon, thereby avoiding modeling the short time-scale earthquakes. We show that this assumption is valid, which implies that the faults that creep and faults with earthquakes display the same time and space evolutions.
\end{abstract}

DOI: 10.1103/PhysRevE.65.056105

PACS number(s): $62.20 . \mathrm{Mk}, 64.60 . \mathrm{Cn}, 81.40 . \mathrm{Np}$

\section{INTRODUCTION}

Geologists have known for over 40 years and materials scientists for somewhat less that if a brittle layer on a plastic substrate is stretched, a state will eventually be reached in which the layer contains system sized cracks that are evenly spaced, with a spacing proportional to the layer thickness. The explanation for this saturated case is trivial: each crack is spaced to avoid the stress relaxation zone of its neighbor, the width of which scales with layer thickness [1]. The deeper question, addressed here, is how does the system evolve from its initial uncracked state to this saturated state.

Geologists have recently observed that the populations of faults (shear cracks) often exhibit power-law length distributions $[2,3]$. It has been suggested that this fault size distribution is what gives rise to the same kind of distribution long observed for earthquakes and known as the Gutenberg-

\footnotetext{
*Now at Exxon-Mobil Upstream Research, Houston, TX; Electronic address: ccspyro@upstream.xomcorp.com

†Electronic address: scholz@1deo.columbia.edu

‡Electronic address: shaw@ldeo.columbia.edu
}

Richter relation $[4,5]$. Such power laws are characteristic of self-organized critical systems [6-10], sometimes considered "universal" states of the system. However, exponential fault size distributions have also been observed [11]. This opens the possibility that these two populations are transition regimes between the end-member states described above. If this is true then, for these systems, power law distributions occupy only a portion of the phase space.

Geological faults grow episodically by frictional stick-slip instabilities, resulting in earthquakes. Here, however, we are interested in the long time-scale phenomena. Let us consider the case of a region of the lithosphere undergoing tension producing deformation of the upper brittle layer, the schizosphere. The schizosphere behaves as a brittle material, and localization of strain causes faults to grow. For time scales of hundreds of thousands of years or longer, the faults nucleate, propagate, and coalesce, organizing themselves in a particular manner. Field data of faults can only serve scientists as snapshots of what state a system is in today. However, a more detailed theory of how systems of faults evolve and why they organize themselves in such states may be possible by studying models that can be set up as simplified cases of geological systems. 
In that spirit we propose a model that explores the different distributions observed during the evolution of a population of cracks as a function of strain. Introducing certain simplifications and assumptions to the problem effectively determines which mechanisms we consider important in producing such behaviors. We assume there are only a few key ways in which the nonlinear interaction of cracks takes place. Agreement of our results with observations makes the case for their importance. The model we introduce in this paper simulates an upper brittle layer coupled to a ductile substrate. We describe a two-dimensional quasistatic system where cracks nucleate and evolve in the brittle layer in response to the deformation of the ductile layer. The displacement field is given as a scalar field in the direction of stretching. The scalar displacement field models tensile cracks. The underlying assumption is that the stress interactions of tensile cracks are similar to those of normal faults. This can be argued by noting that in the planar view the stress field around the tips of a normal fault (mode III shear crack) are exactly symmetrical to those of tension cracks. Although the stress in the case of normal faults is due to shearing, whereas in the case of tension cracks it is due to tension, the interaction of these symmetrical fields around cracks is what we are interested in capturing. The physics determining the behavior of the system studied here may be summarized as follows.

(a) The friction law of the cracks. This model mimics faulting on the crust. In order to get localization of strain an important physical mechanism is slip weakening, which renders an already cracked surface weaker than before and thus more likely to slip again as more strain is loaded into the system. The crack tips have a much higher stress concentration than the area around them and the crack propagates as a response to further extension of the bottom layer. The slipweakening function used in this paper is modeled as an initially linear decay of yield strength of the brittle material. We use linear stability analysis to study the parameter space of slip weakening, and we find a wide range of values for which the system is stable. This parameter space has a second variable, the rheology of the ductile layer, which is the next physical parameter in the problem.

(b) The constitutive law of the two layer interface. In the case of this model we study two-dimensional (2D) growth of cracks that are as deep as the top layer. The ductile substrate is simulated as a plastic layer with strain hardening. However, as we will see, we will be able to scale this part of the problem out.

(c) The heterogeneity of the brittle layer. The crust can be thought of as a brittle layer of variable yield strength due to imperfections or variations such as inclusions, prior ruptures, variable material composition, etc. This disorder is modeled here as a randomly distributed initial yield strength threshold of the brittle layer. This aspect of the model resembles previous work done by Colina, de Arcangelis, and Roux [12] using an electric analog of a layer of fuses coupled by resistors to a bottom layer where a uniform electric field was imposed. They varied the heterogeneity introduced in the system and looked at the effects it produced to their system. Similarly we also observe that the number of cracks obtained for a given strain depends on the disorder. For very homo- geneous systems and at low strain, slip localizes mostly on large evenly spaced cracks, while for more heterogeneous systems at the same strain, slip gets distributed over a range of sizes of cracks.

Given these parameters, we found that the growth of populations of cracks is characterized by three regimes as a function of strain, which have three distinct crack size distributions. Initially, as the system is strained, we observe an increasing number of new cracks. This regime is dominated by the heterogeneities, and leads to an exponential distribution. This is the nucleation regime. With further stain, when the slip weakening effects become comparable to the disorder, the cracks begin to grow, and the distribution approaches a power law. This is the growth regime. As strain continues, more cracks are coalescing to form longer cracks than there are cracks nucleating, and therefore the number of active cracks begins to decrease. The power law becomes distorted, with the largest cracks approaching an exponential distribution. This is the coalescence regime. Finally, for even higher strains the system organizes into the maximum number of cracks it can hold for any additional strain. This is the saturation regime. At this stage the cracks have grown into a pattern of long arrays that are spaced apart proportionally to the depth of the brittle layer, with the largest cracks having an exponential distribution. Figure 1 is a simulation using the above model, which shows a snapshot of a network of cracks in the planar view at increasing strain. In the sections to follow we study these populations and explain their interaction mechanisms.

We have organized the sections as follows. In Sec. II we describe the model. Section III has the numerical results and the stability analysis with Sec. IV briefly comparing these results to observations. The last section contains our conclusions.

\section{THE MODEL}

We want to study the problem of the crack population formation and its evolution on a brittle layer that is driven on the bottom by an extending layer. First, the lower plastic layer is extended by a small amount. That in turn strains the top layer whose equilibrium requirement is satisfied when the total stresses applied to it are lower than its yield strength. If at any point on the brittle layer the yield strength is exceeded, a crack is allowed to form with slip opening $h$. The crack accumulates slip until the stress on it satisfies the boundary condition, in other words the stress is less than its yield strength. Once that condition is satisfied, the system has reached quasistatic equilibrium. It gets driven by additional extension applied to the bottom layer, and the process repeats itself.

Figure 2 shows a schematic representation of the discretized model. It is a two-dimensional system of spring blocks with the $x$ and $y$ dimensions scaled by the thickness of the top layer. The resolution in the plane can be effectively changed by varying the spring constants $k_{x}$ and $k_{y}$. All lengths in the problem scale with $L$, the width of the brittle layer. We therefore approximate the $3 \mathrm{D}$ problem as a $2 \mathrm{D}$ problem. It is reasonable to expect that the in-depth slip profile of cracks is important in examining their growth rates and their shapes. The representation of the vertical stress by 

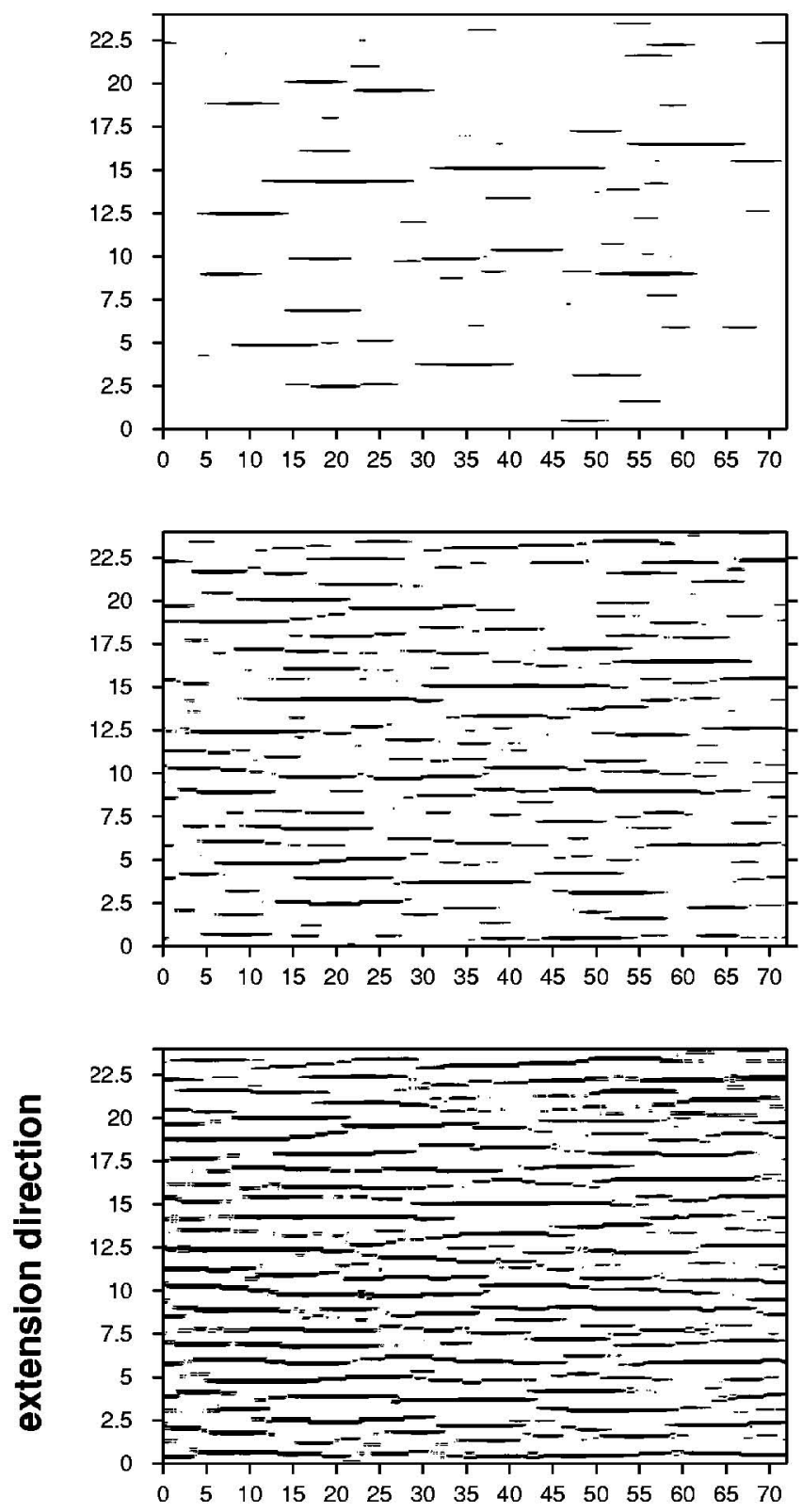

\section{distance along faults}

FIG. 1. Snapshot of model simulation shows plan view of a network of cracks at different strain values. The axes are scaled by the brittle layer thickness. The cracks propagate along the axis normal to the direction of extension. Crack evolution with strain $\epsilon$. (a) $\epsilon=0.064$, (b) $\epsilon=0.072$, (c) $\epsilon=0.08$.

the leaf springs is an approximation of the fracture energy $G$ of a crack growing in three dimensions. Of course, this function oversimplifies the rheological behavior of real material interfaces, which is much more complicated but has no known analytical form.

Another simplification in the model is the use of a scalar displacement in the direction parallel to that of the loading. This is an acceptable first-order approximation to the displacement field for the case of uniform extension. An important ingredient of this model is the dynamics introduced

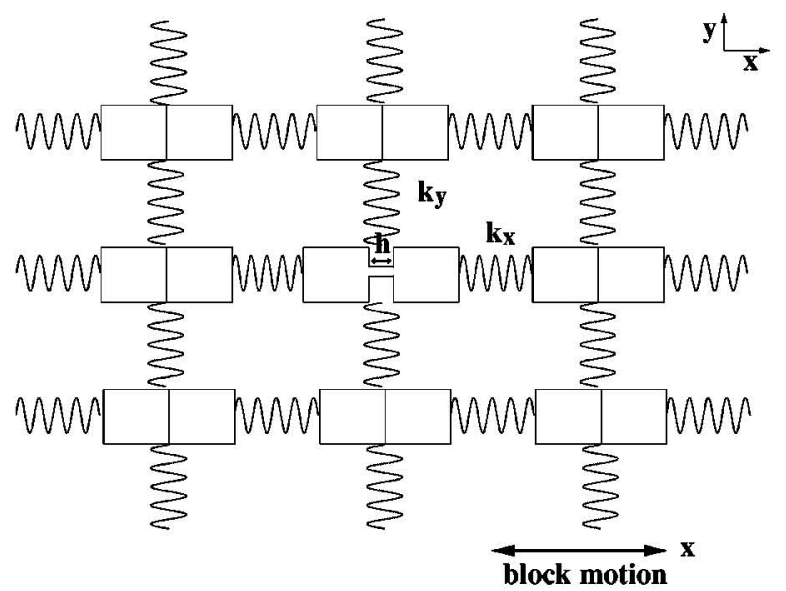

Top View

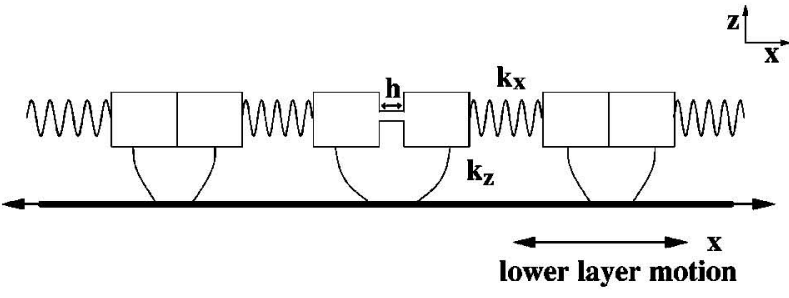

Side View

FIG. 2. Schematic representation of the model. Looking from the top, there are coil springs in the $y$ direction and coil springs in the $x$ direction. The blocks can move in the $x$ direction only. A crack is shown as an opening of a certain distance $h_{i j}$ in the middle of block $(i, j)$. Looking from the side, there are leaf springs in the $z$ direction such that the spring coefficient ratios $k_{x} / k_{z}$ and $k_{y} / k_{z}$ set the resolution scale. The lower ductile layer is stretched and drives the upper brittle layer through the leaf springs. (The extension direction $x$ is rotated $90^{\circ}$ in this figure with respect to Fig. 1).

through the slip weakening friction law. The slip weakening law drives much of the localization of strain on the surface and that, in turn, leads to avalanches of cracking events $[13,14]$. The effects of this friction law will be further analyzed in the following section. Another feature of this model is the coupling of the top layer to a bottom layer through the propagation resistance stress. While we have posed the problem so that we can study different rheologies, we will focus here on the small disorder limit where the different rheologies can be effectively scaled out of the problem.

The dynamic 2D scalar model for the brittle layer we examine is given by the Klein-Gordon equation

$$
\frac{1}{c^{2}} \frac{\partial^{2} u}{\partial t^{2}}=\frac{\partial^{2} u}{\partial x^{2}}+\frac{\partial^{2} u}{\partial y^{2}}-\frac{(u-U)}{L^{2}}
$$

where $u$ is the displacement, $t$ is the time, $x$ and $y$ are the perpendicular directions, $U$ is the displacement of the lower ductile layer, $c$ is the wave speed, and $L$ is the brittle layer depth. The first three terms are from the wave equation, our scalar approximation of the linear elasticity of the stress in the horizontal direction. The last term is a linearized approxi- 
mation of the stress in the vertical direction that couples the two layers. The bottom layer has a constant (homogeneous) strain boundary condition,

$$
\frac{\partial^{2} U}{\partial x \partial t}=\nu
$$

We use periodic boundary conditions in the $x$ and $y$ directions.

We want to study the problem of crack growth, which has a much larger time scale than that of wave propagation, (i.e., earthquakes will not be treated in this paper). Therefore, we hypothesize that the propagation and organization of faults are long time-scale phenomena, which can be approximated by letting the wave speed, i.e., the speed of earthquake propagation, approach infinity. Separation of these two time scales, where $c \rightarrow \infty$, gives the following Poisson problem:

$$
\frac{\partial^{2} u}{\partial x^{2}}+\frac{\partial^{2} u}{\partial y^{2}}-\frac{(u-U)}{L^{2}}=0
$$

In order to study the originally proposed model of a brittle layer driven by an elastic bottom layer, we must construct the top layer with brittle properties. This will be very simply described by introducing a yield strength threshold boundary condition. The yield strength is constructed as

$$
\Phi= \begin{cases}\Phi_{0}, & h=0, \\ \Phi_{0}+m h-\frac{\tilde{\alpha} h}{1+\tilde{\alpha} h}, & h>0,\end{cases}
$$

where $\tilde{\alpha}>0$ and $m>0$. We define $h$ to be the integrated strain across a discontinuous boundary, which is given as the following limit:

$$
h(x)=\lim _{\epsilon \rightarrow 0} \int_{x}^{x+\epsilon} \frac{\partial u}{\partial x} d x .
$$

In the case where there is no crack, the yield threshold is just given by $\Phi_{0}(x, y)$ as a random distribution of strength. In the case where there is a crack, the yield strength includes a slip weakening friction law with no time healing where $\Phi_{0}(x, y)$ is the stochastic part of the function given at $t=0$.

Our choice of the slip weakening function for the strength of the material is meant to capture the essential physics, though it is a huge simplification of all that occurs in material failure. The biggest simplification is that here we consider fracture energies associated only with interfaces, while there are bulk effects associated with process zones $[3,15]$ and plastic deformation [16], which may be important as well. Our main justifications for our simplification is that the slip weakening is generally considered a central component of the breakdown process, and that it makes the problem much more tractable and computationally efficient, and captures well the observations, as we will see. Moreover, it is only a linearization of the slip weakening that dominates the model behavior we examine here.

The equations of motion are symmetric with respect to the addition of a constant to $U$ and $\Phi_{0}$, so only differences in the values of $u$ are relevant to the dynamics of the system. The parameter $m$ determines an effective rheology in the coupling of the two layers. For small $h$ we can simplify the equation of the yield strength by incorporating the strain hardening rheology in $\widetilde{\alpha}$. Therefore, we can study a collective effect in the slip weakening parameter by defining $\alpha$ $=\tilde{\alpha}-m$,

$$
\Phi=\Phi_{0}-\frac{\alpha h}{1+\alpha h}, \quad h \geqslant 0,
$$

an approximation of Eq. (4) valid to the linear order. For small $h$, we could drop the denominator altogether, but we keep it here in Eq. (6) so as to keep the strength formulation physical for all values of $h$. Thus, we can write the boundary condition along a cracked interface in static equilibrium as

$$
\frac{1}{2}\left(\left.\frac{\partial u}{\partial x}\right|_{x=\text { crack }^{-}}+\left.\frac{\partial u}{\partial x}\right|_{x=\text { crack }^{+}}\right) \leqslant \Phi_{0}-\frac{\alpha h}{1+\alpha h},
$$

where the stresses on that point have to be equal to or less than the yield strength. If the stresses at any point exceed the yield threshold, then the crack slips more until the boundary condition is satisfied. In this paper we show that these effective parameters alone can capture many of the most interesting features of crack networks. The next point we have to address is how $h$ gets updated. We assumed that the cracks relax to a quasistatic steady state faster than the loading rate, $\nu$ and that $\partial h / \partial t$ does not affect the way the cracks will develop and their organization. We test this hypothesis numerically, and in the following section we show that it holds true.

To discretize the model, it is convenient to make a change of coordinates. We consider the variable $w_{i j}=u_{i j}-U_{i j}$ representing the displacement of the upper layer relative to the lower layer evaluated at each node; a crack, when it forms, is taken to reside at the center of the node and has the opening $h_{i j}$ at that node. Thus, the displacement just to the right of a crack of width $h_{i j}$ is $u_{i j}^{+}=w_{i j}+h_{i j} / 2+U_{i j}$ while the displacement just on the other side is $u_{i j}=w_{i j}-h_{i j} / 2+U_{i j}$. In these variables, the equation of the model is

$$
\begin{gathered}
k_{x}\left(w_{i+1 j}-2 w_{i j}+w_{i-1 j}\right)+k_{y}\left(w_{i j+1}-2 w_{i j}+w_{i j-1}\right) \\
+\frac{k_{x}}{2}\left(h_{i+1 j}-h_{i-1 j}\right)-k_{z} w_{i j}=0
\end{gathered}
$$

with $i=1 \rightarrow N_{x}$ and $j=1 \rightarrow N_{y} ; N_{x}$ and $N_{y}$ are the number of grid points in the $x$ and $y$ directions. This is the discrete version of Eq. (3) with $k_{x} \equiv 1 / \Delta x^{2}, k_{y} \equiv 1 / \Delta y^{2}, k_{z} \equiv 1 / L_{2}$. The square lattice used here for the scalar field of the displacement introduces an inherent anisotropy to the model. However, this effect is rather inconsequential in the realm of this study since we have tensile straining of the material, which is in itself anisotropic, leading to surface cracks that propagate mostly in the direction normal to that of the extensional force.

The updating of the system occurs as follows. First a small strain step $\nu \delta t$ is made, then the stresses $\sigma_{i j}$ are cal- 
culated for all nodes. If the stresses are above threshold then $h$ gets updated. The slip displacement of the cracks, $h$, get updated by adding small $\Delta h_{i j}$ increments to it, and recalculating the stresses on the brittle layer until the equilibrium equation is satisfied. The equation of evolution of $h$ is

$$
h_{i j}^{n+1}=h_{i j}^{n}+\Delta h_{i j},
$$

where $n$ is the relaxation step of the time scale of earthquakes. There are various ways of updating $h$. Here we present two different functions for calculating $\Delta h_{i j}$. One way is to compute it as a percentage of the stress drop and the other is to compute it as a constant step increment.

$$
\Delta h_{i j}= \begin{cases}p \frac{\Phi_{i j}}{k_{x}}\left(\frac{\sigma_{i j}}{\Phi_{i j}}-1\right), & \frac{\sigma_{i j}}{\Phi_{i j}} \geqslant 1, \\ 0, & \frac{\sigma_{i j}}{\Phi_{i j}}<1,\end{cases}
$$

or

$$
\Delta h_{i j}= \begin{cases}\Delta h, & \frac{\sigma_{i j}}{\Phi_{i j}} \geqslant 1, \\ 0, & \frac{\sigma_{i j}}{\Phi_{i j}}<1 .\end{cases}
$$

$p$ takes a value from $0<p<1$ and is a percentage of the stress drop. Naturally the question to ask is: how do the results depend on the two choices of functions? As we will see, they do not.

\section{RESULTS AND ANALYSIS OF THE MODEL}

In this section we shall discuss the linear stability analysis as well as present the results obtained by isolating and combining the parameters, treating first the irrelevant, and then the relevant parameters to the dynamics.

\section{A. Linear stability analysis}

Let us first consider what happens with the slipweakening instability we have introduced in the model. A linear stability analysis of the growth of Fourier modes will show for which values of $\alpha$ the instability will grow. Starting from the wave equation, let us expand about $u=U$,

$$
\frac{\partial^{2} u}{\partial x^{2}}+\frac{\partial^{2} u}{\partial y^{2}}-\frac{u}{L^{2}}=\frac{1}{c^{2}} \frac{\partial^{2} u}{\partial t^{2}}
$$

and now the boundary condition is

$$
\left.\frac{\partial u}{\partial x}\right|_{x=\text { crack }}=-\frac{\alpha h}{1+\alpha h} .
$$

$\Phi_{0}$ does not appear in the equation since it is a first-order term that satisfies the boundary condition of the mean displacement. Linearizing the equation of a Fourier mode exp $\left(-k_{x} x+i k_{y} y+\Omega t\right)$ we get a dispersion relation of

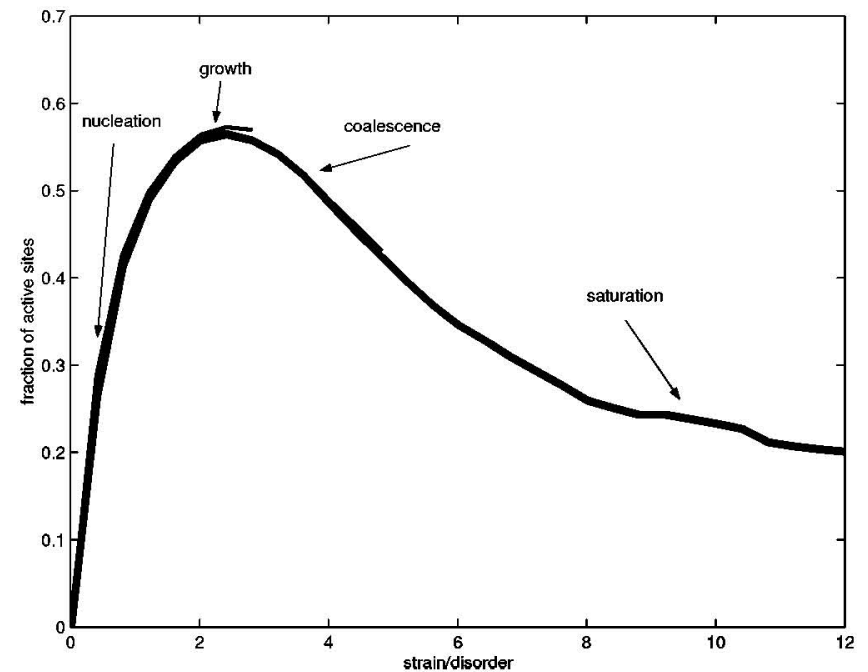

FIG. 3. Fraction of active sites evolving with increased strain. On the horizontal axis, excess strain $\varepsilon \equiv\left(\nu t-\Phi_{0}\right) / g$ is normalized by disorder. Disorder $g=10^{-4}, 10^{-3}, 10^{-2}$, with thicker lines corresponding to smaller $g$. Note the overlaying of curves. The three different strain regimes are labeled.

$$
\Omega= \pm c \sqrt{\left(\alpha^{2}-k_{y}^{2}-\frac{1}{L^{2}}\right)} .
$$

For stability we need $\Omega$ to be imaginary, i.e., $\alpha^{2}<k_{y}^{2}$ $+1 / L^{2}$. When cracks nucleate, the wave number $k_{y}$ is very large, so the restriction on $\alpha$ is easily satisfied, whereas for very long cracks $k_{y}$ approaches zero and in order to keep the stability we must have $\alpha<1 / L^{2}$. This imposes an upper bound for $\alpha$. A lower bound is given from the fact that for the slip weakening to localize strain, it must at least beat the strain hardening rheology. This means that $\tilde{\alpha}-m>0$ and therefore, $0<\alpha<1 / L^{2}$. In the following simulations we have set $L \equiv 1$, without loss of generality, in which case the bounds on $\alpha$ are $0<\alpha<1$. We distinguish between two types of parameters: those that are irrelevant or can be scaled out, and those that remain relevant. We look at each in turn.

\section{B. Irrelevant parameters}

For large enough systems, the system size is irrelevant. For the distribution of breaking strengths, we let $\Phi_{0}(x, y)$ $=\Phi_{0}+g \xi(x, y)$ where $\xi$ is a random number between 0 and 1 . For small disorder, when $g \ll 1$, a number of parameters can be scaled out. First, we will see a number of regimes as strain is increased, but all of them, for $g$ small, occur when $h \ll 1$. Thus, the earlier approximations of absorbing the rheology into $\alpha$ is valid, and we only need to consider the one parameter $\alpha$ for the strength evolution. A second scaling can be made to remove $g$ itself: by dividing the strain increment above $\Phi_{0}$ by $g$, we get the brittle strain excess $\varepsilon \equiv(\nu t$ $\left.-\Phi_{0}\right) / g$ which then collapses the effective loading for $g$ ranging over many orders of magnitude. Figure 3 illustrates this result through the overlay of a number of curves with different values of $g$. Figure 3 shows the fraction of active sites as a function of $\varepsilon$, for $g$ ranging from $10^{-5}$ to $10^{-2}$. 


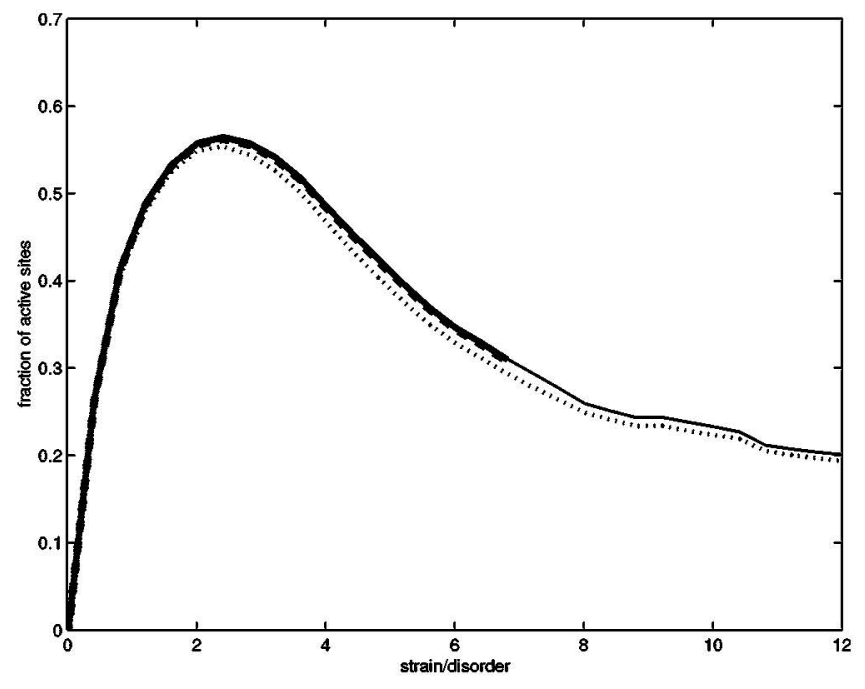

FIG. 4. Number of cracks per unit area as a function of strain, $\varepsilon$, for four different cases of updating the slip displacement $h$ at each strain step. The relaxation is done using slow increments of the displacement $h$ such that the total stress on the crack is less than its yield threshold. For the first two cases, we let the increments be a fraction of the stress above threshold; these cases are shown by the two thick lines, with the solid line having a smaller fractional increment compared to the dashed line. For the other two cases, we take constant $\delta h$ strain opening steps; here again the solid thin line has a smaller increment relative to the dashed thin line. Observe that the curves all basically overlay.

Active sites are the sites that have broken recently. We consider sites that have broken recently, as we are interested here in what part of the network is currently accommodating strain. For all cases we observe the following: at the beginning the fraction of active sites increases with elastic strain. This indicates that initially nucleation is the dominant process. When the slip displacement on the crack is big enough for slip weakening to become comparable to the disorder, the slip weakening begins to dominate, and the slip localizes so that the number of active sites starts to decrease; this is the growth regime. The system then transitions to a regime of coalescence of cracks where the rate of coalescence dominates that of the nucleation. Eventually, the fraction of active sites asymptotes to the saturated regime where we get cracks that are evenly spaced about one layer thickness apart. These four regimes are central to the behaviors for the general parameter space of the model, and we will return to them when we later examine the distribution of sizes of cracks.

Grid resolution does not play a role at the large scales, and we find stable distributions of crack sizes for sufficiently resolved grids. Event dynamics, through the relieving of stress via the updating of $h$, is also irrelevant. Figure 4 illustrates this with a range of choices of parameters $p$ and $\Delta h$ in Eqs. (10) and (11). The first two cases shown are with fraction updates of stress excess above threshold, from Eq. (10), with the fraction $p$ taking the values 0.4 and 0.7 . The two other cases shown are with constant slip increments, from Eq. (11), where the increment $\Delta h$ was equal to $10^{-6}$ and $10^{-5}$. The figure shows that, for all the different ways of updating $h$, the three regimes of evolution of cracks are al- most identical. This means that the details of $\partial h / \partial t$ are not important for relaxing the system to its quasistatic equilibrium within each loading step. This validates our hypothesis that we can study the growth of faults in time and space without necessarily modeling the earthquakes. Faults that grow by earthquakes and faults that creep, all else being equal, have indistinguishable spatial properties. Thus, we are left with one fundamental relevant parameter, the net slip weakening $\alpha$.

\section{Slip weakening}

The linear stability analysis above shows that the equation for the crack evolution has a stable regime for $\alpha$ between 0 and 1 . In the case of $\alpha=0$, we expect to get no localization of deformation into cracks. The dynamics are controlled solely by the randomly distributed yield threshold at $t=0$, so each site will crack according to that distribution and the slip localization on the cracks remains uncorrelated. For the case of $\alpha=1$, we have marginal stability where a crack could grow for very small strains. The crack will keep weakening the more it slips since its friction is a function of the slip displacement. For $\alpha>1$, it will be unstable in the sense that there will be no quasistatic equilibrium solution of $h$ such that the boundary condition is satisfied. We get a runaway crack as the more it slips to satisfy the threshold condition the more it weakens.

Figure 5 shows six simulations done with different $\alpha$ values. These are snapshots of a part of the grid at given strains. The figure is organized in order of decreasing $\alpha$ from 1 to 0 . We show the particular six values, because they are indicative of the different behaviors of the model. Figures 5(a) and 5(b) are shown at lower strains than the rest, because they are at or near the upper limit and any higher loading causes catastrophic failure. It is clear that when $\alpha \rightarrow 1.0$, all the deformation localizes onto few cracks which soon grow unstable. For intermediate values of $\alpha$, Figs. 5(c) and 5(d) the cracks are stable and their number increases while they keep the separation distance of one depth layer between large cracks. As $\alpha \rightarrow 0$, Figs. 5(e) and 5(f), the deformation does not localize, and we do not get a full spectrum of sizes of cracks.

Before continuing, we should mention how we define a crack. We take a crack to be the network of all the nodes that have touching neighbors. We can vary the allowed neighbors as either the four nearest nodes or only the two collinear nodes in the direction of propagation. So, if two crack tips are offset by one grid point in the diagonal [e.g., $(i, j)$ and $(i+1, j+1)]$, they are not counted as one crack but as two separate ones. We also keep track of one other feature of crack openings, which is when it last opened. In this way, we can distinguish between active and inactive sites. This is important, because as strain accumulates, more and more sites are cracked, and we eventually run into a percolationlike behavior if we consider the connectivity of all sites that have ever cracked. This leads to sensitivity in how we define connectedness and cracks. If, instead, we consider only the sites that have broken recently, we find well behaved distributions of cracks, which are insensitive to choices (such as how re- 
displacement

(a)

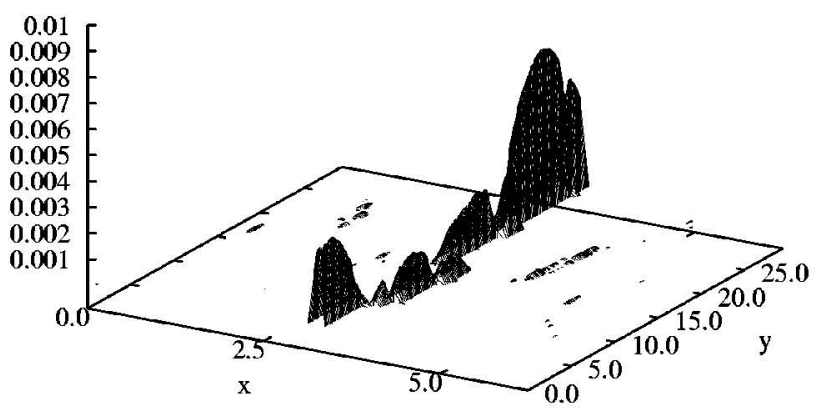

displacement

(c)

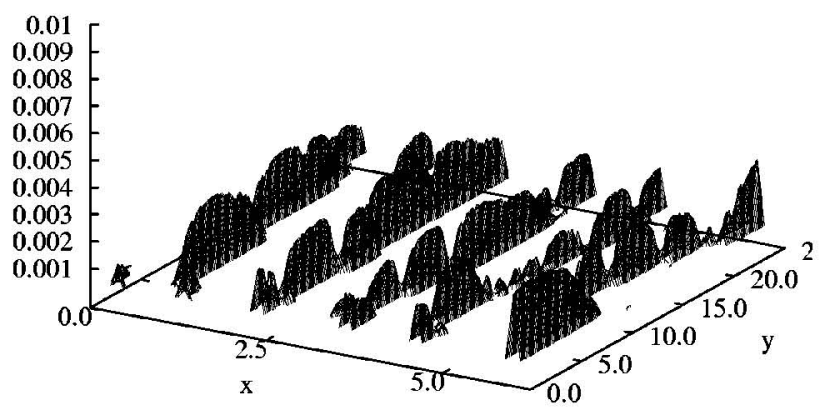

(d)

displacement

(e)

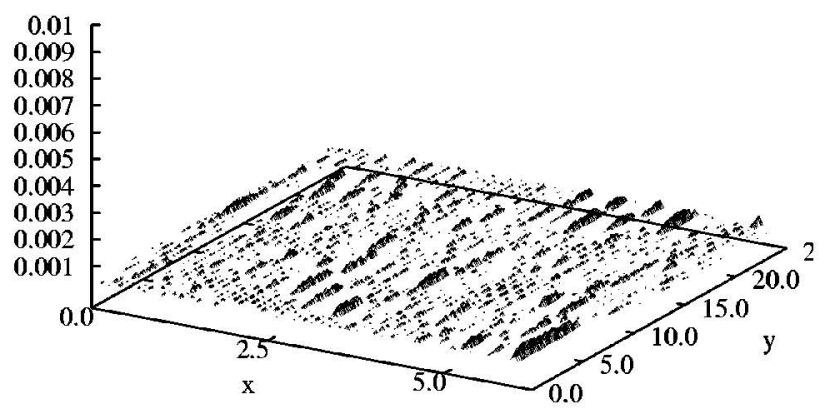

displacement

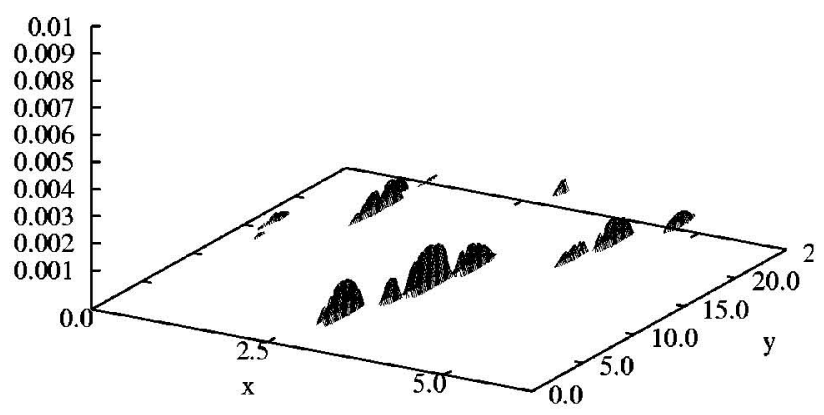

displacement

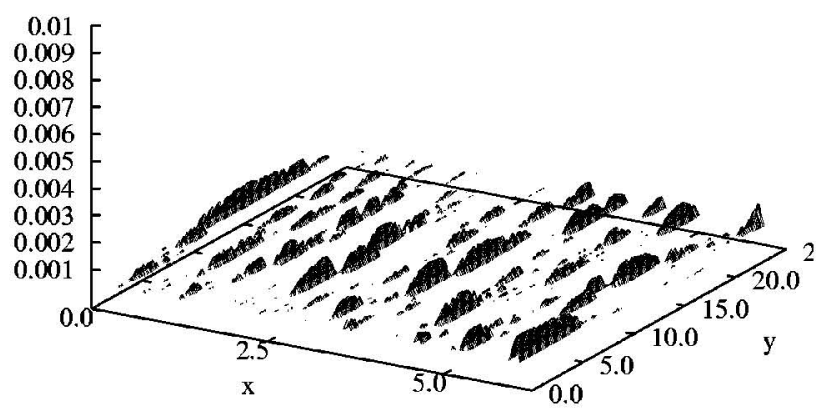

displacement

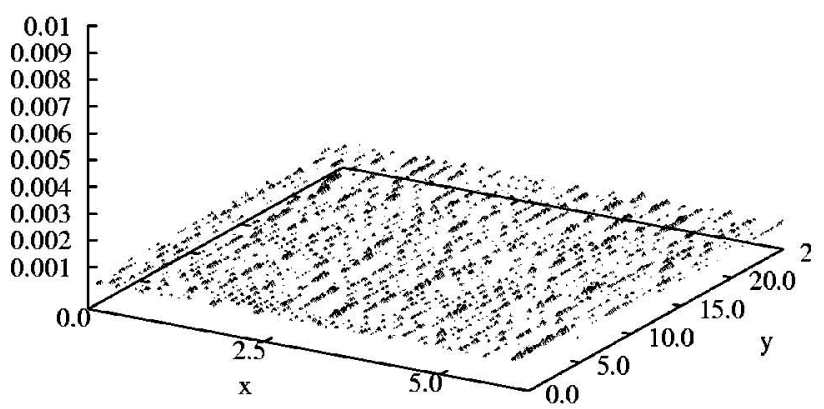

FIG. 5. Oblique angle view of simulations for different slip weakening values, $\alpha . x$ axis is scaled by the brittle layer thickness. Slip displacement is ploted on the $z$ axis. (a) $\alpha=1.0, \epsilon=0.02 \%$, and (b) $\alpha=0.9, \epsilon=0.07 \%$; cracks grow unstable for additional strain, $\epsilon$. (c) $\alpha=0.4, \epsilon=0.19 \%$. The system evolves in a stable manner. (d) $\alpha=0.2, \epsilon=0.19 \%$. (e) $\alpha=0.1, \epsilon=0.19 \%$. Deformation is less localized. (f) $\alpha=0.0, \epsilon=0.19 \%$. There is no localization of deformation.

cently they have broken). Thus, when we speak of cracks, we speak of connected elements that have broken within the last time (equivalently strain) interval $\Delta \varepsilon$.

Figure 6 shows the fraction of active sites as a function of the elastic strain as we vary $\alpha$. Active sites are the sites that have broken recently. There are four data sets altogether, showing different values of $\alpha$. We see, as described before, four different regimes, the nucleation, the growth, the coalescence, and the saturation. As $\alpha$ decreases, we observe that the maximum fraction of active sites increases while it shifts towards larger strains.

Let us now examine the distribution of active crack sizes in the different regimes. Here we use a fixed value of $\alpha, \alpha$ $=0.2$. Figure 7 shows the distribution of crack lengths, plot- ted on a log-linear scale, for small loading. We see exponential distributions of lengths for the very smallest loading, with slopes decreasing as the fraction of active sites increases. Towards the end of this regime, we see crack lengths exceeding the extrapolated exponential distribution, as the upward curvature indicates. These distributions approach a power law, as Fig. 8 shows, plotting now on a log-log scale, for the full range of loading. Here we see the exponential distributions for low loading falling off, then the approach to a power-law, with slope around -2 , indicated by the dashed line. At the highest values of strain, the largest sizes appear to asymptote, approaching a bump on this plot, while the smaller sizes remain power-law-like, though somewhat diminished in number. Figure 9 probes this largest size distri- 


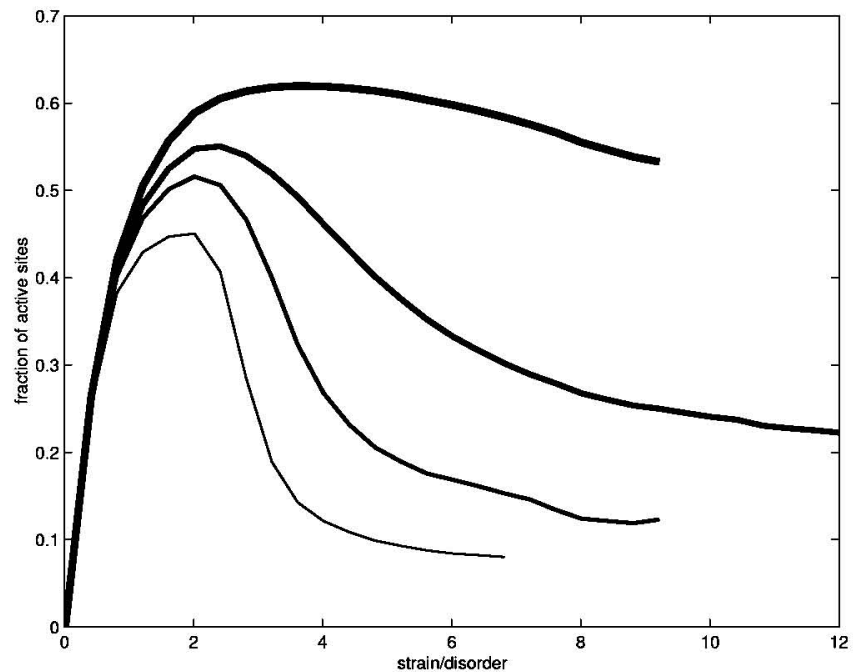

FIG. 6. Fraction of active sites as a function of strain, $\varepsilon$, for four different values of slip weakening $\alpha$. In all four cases shown there is a nucleation regime (the number of cracks increases with strain), the growth regime (the number of cracks peaks), a coalescence regime (the number of cracks starts to decrease), and a saturation regime (constant density of cracks spaced one layer thickness apart). As $\alpha$ decreases, the maximum number of cracks per unit area increases while it shifts towards larger strains. Weakening $\alpha=0.1$, $0.2,0.3,0.4$. Thicker lines correspond to smaller $\alpha$.

bution, this time plotted on a log-linear scale. We see here then the clear exponential distribution of these largest cracks in the high strain saturation regime.

This behavior is the same for other values of $\alpha$, with only some quantitative differences. Figure 10 shows the relevant distribution curves for $\alpha=0.4$, indicating a similar powerlaw slope value for the growth regime, with a slightly steeper but nevertheless exponential distribution for the largest cracks in the saturation regime.

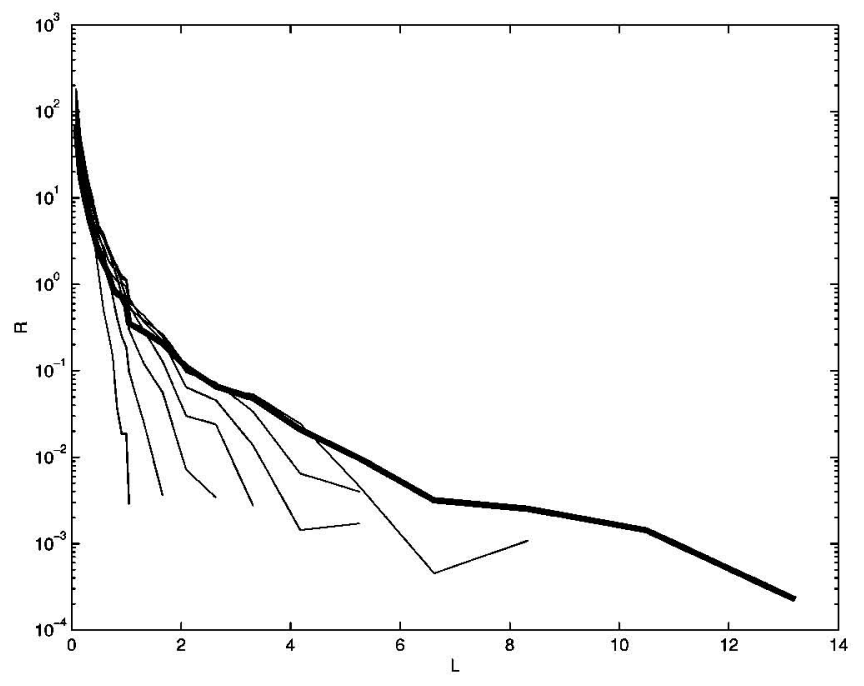

FIG. 7. Log-linear plot of distribution of sizes for increased strain, at low strain values in the nucleation regime. Strain increases from left to right, with the highest strain indicated by the thick line. Note the initially exponential distribution.

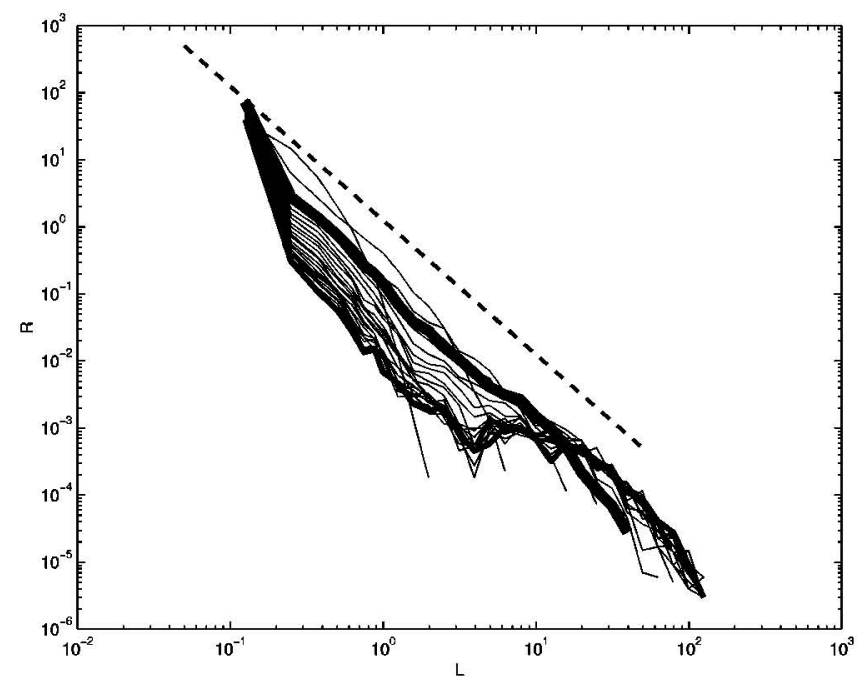

FIG. 8. Log-log plot of distribution of sizes for increased strain. Note the power-law distribution at intermediate strain, indicated by the thickest line, and the asymptotic distribution at higher strain, indicated by the other thick line. The dashed line shows a slope of -2 for comparison.

\section{COMPARISON WITH DATA AND A PHYSICAL MODEL}

Geologic data from normal faults show the main features we have discussed in the paper. Fig. 11(a) shows data from the surface of Venus where normal faults were radar imaged during the Magellan mission in 1989 [17-19]. SAR mapping of 1750 faults on the low plains of Venus, which have undergone extension, reveals a clear power-law scaling of frequency-size distribution with an exponent of -2.02 . The resolution of that image was $70 \mathrm{~m}$ per pixel and the scanned area was about $10^{4} \mathrm{~km}^{2}$. The total strain for the Fig. 11(a) dataset is calculated to be around $1 \%$.

Figure 11(b) is a log-linear plot of the frequency-size distributions of normal faults sonar imaged from the flanks of mid-ocean ridges. Two different strains are shown, namely, $5 \%$ and $10 \%$, the second being an order of magnitude larger than in Fig. 11(a). The distributions observed here are clearly exponential. This is a distinct regime in the fault growth, which would correspond to the coalescence regime of our model. Recently, we have demonstrated the strain transition from power law to exponential fault distributions in a single geological site [21]. Finally, Fig. 11(c) shows the distance between tensile cracks as it scales with the thickness of the bed they are in. The two kinds of points indicate two different rock types, and they both show a linear scaling. This graph corresponds to the saturation regime where we observe that the system size cracks about a thickness layer apart.

Finally, in a physical model in which tensile cracks form in a thin clay layer stretched on a rubber sheet, we observed the same crack population history as Fig. 3, together with the corresponding transition from power law to exponential size distributions [22].

\section{CONCLUSIONS}

In this paper we have shown that a model with only slipweakening instability, strain-hardening rheology, and ran- 


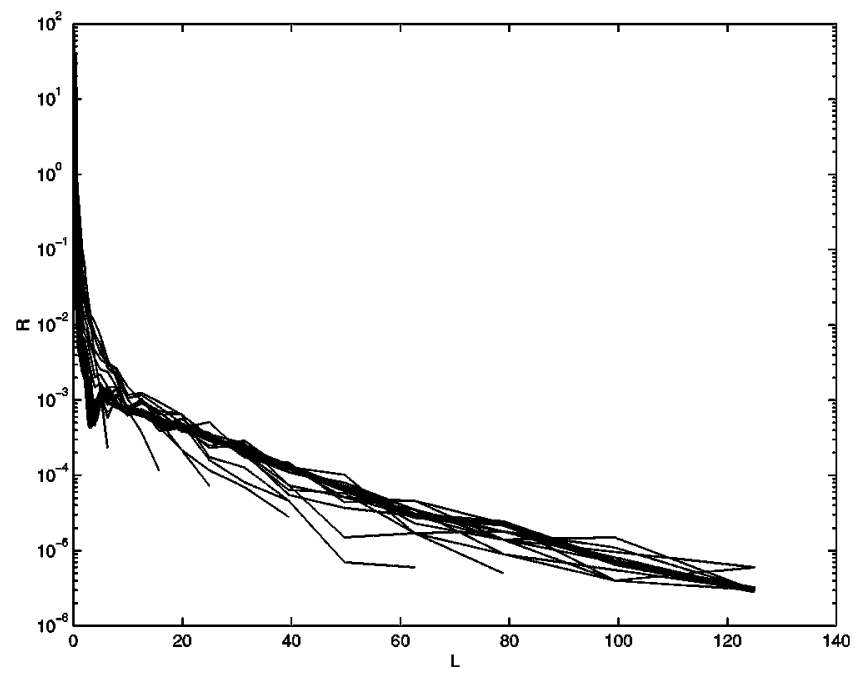

FIG. 9. Log-linear plot of distribution of sizes for increased strain, at large strain values. Note the exponential distribution of largest events at large strains. The highest strain is highlighted with the thicker line.

(a)
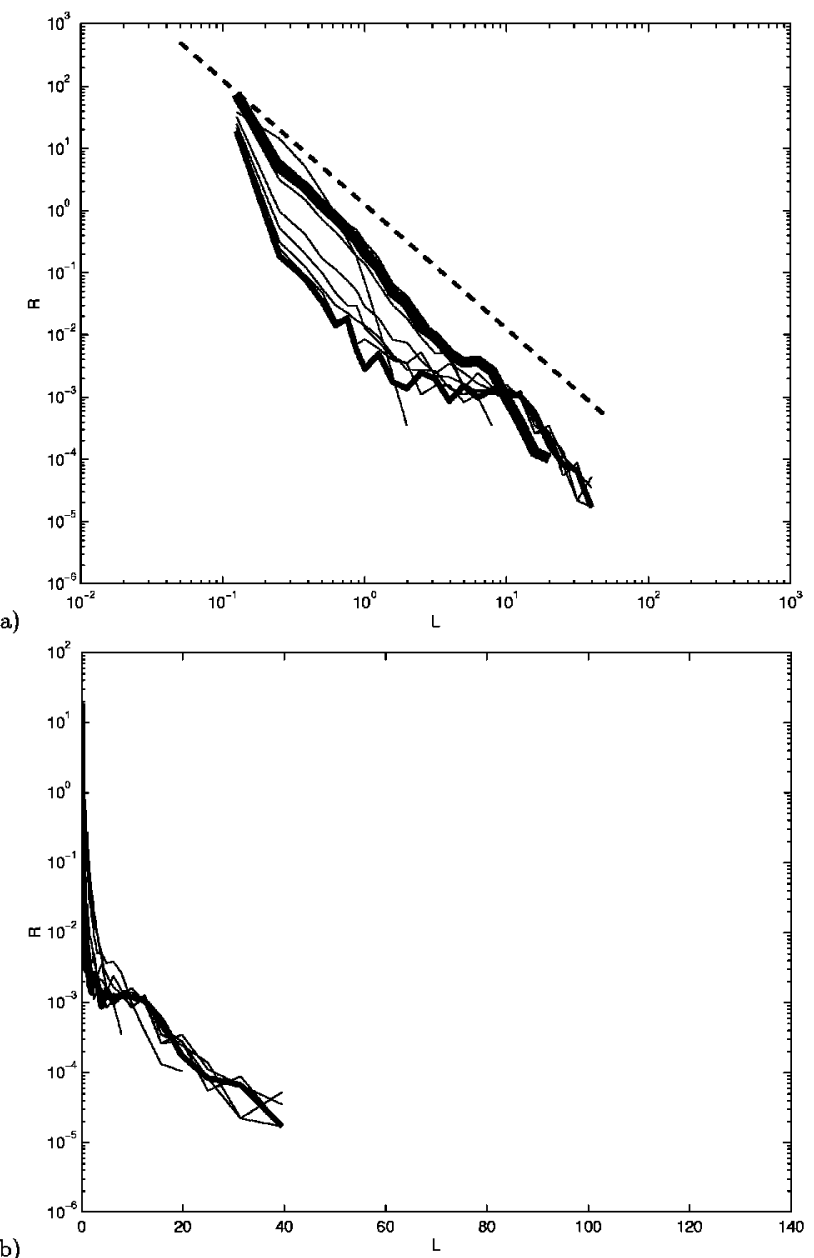

FIG. 10. Distribution of sizes of cracks for larger value of the weakening, $\alpha=0.4$. (a) Log-log plot; note the similarity with Fig. 8 (b) Log-linear plot; note again the exponential distribution at large strain, as in Fig. 9, but here with a higher slope.

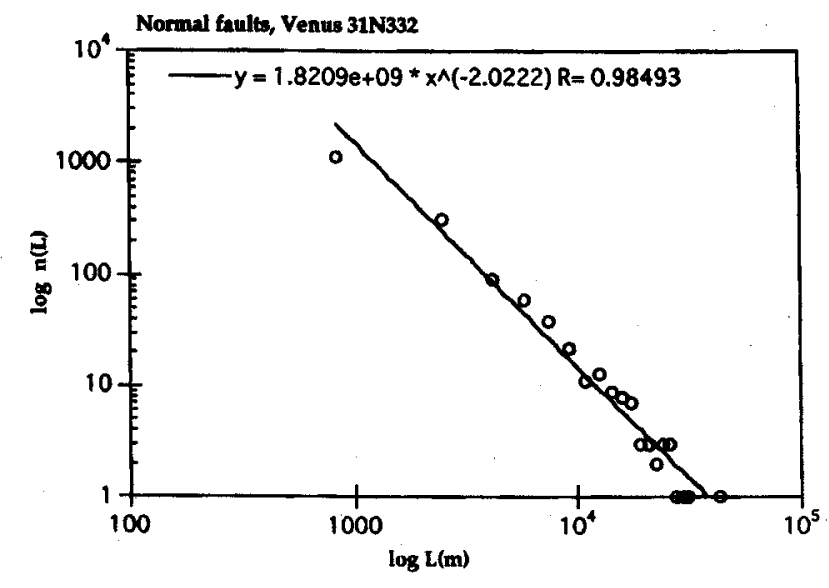

(a)

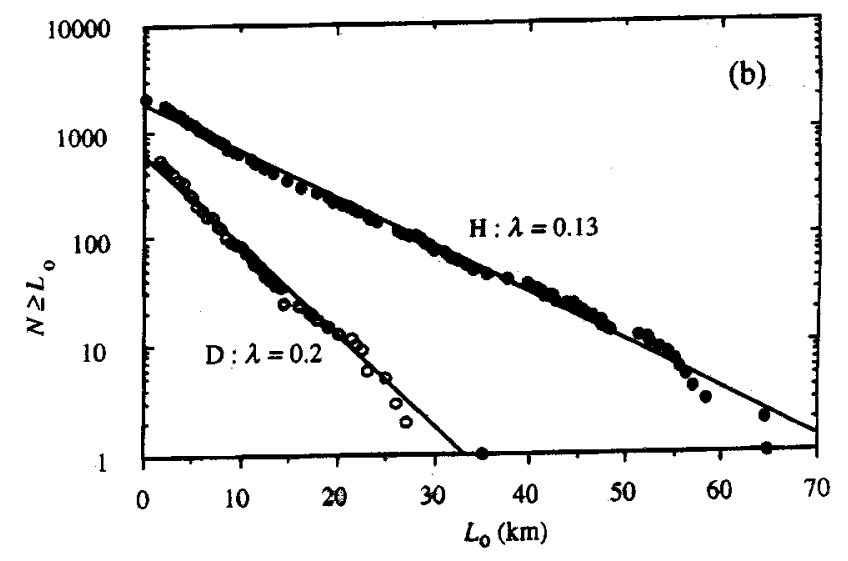

(b)

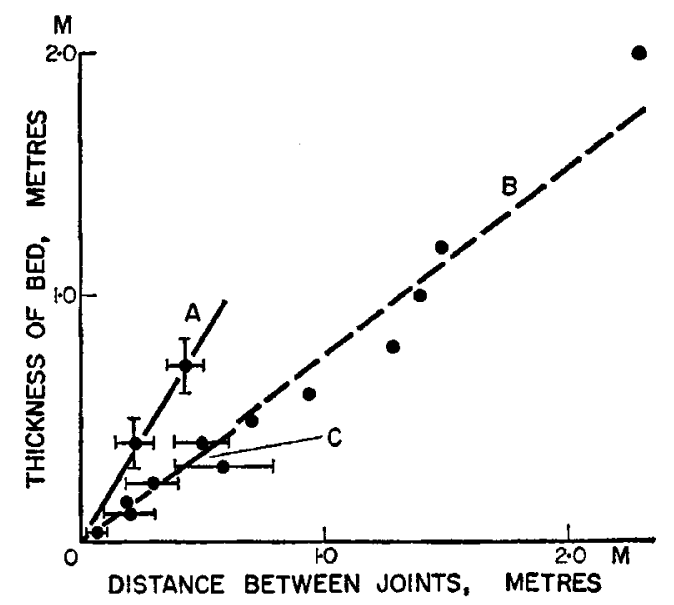

(c)

FIG. 11. Geological evidence of the three evolution regimes. (a) Power-law distribution (exponent is -2.02) for normal faults on Venus. Strain is around 1\%. Figure from Scholz, 1997 [4]. (b) Loglinear plot shows exponential frequency-size distributions for midocean normal faults at $5 \%$ and $10 \%$ strains. Figure from Cowie et al., 1993 [11]. (c) Tensile crack spacing scales with the layer thickness. The two point types are for two different types of rock. This saturation regime has system-size cracks, one thickness layer apart. Figure from Price; data from Bogdanov, Kirollova, and Novikova $[20]$. 
domly distributed thresholds of yield strength can be used to study the evolution of a population of cracks in time and space. For very strong slip weakening, $\alpha>1$, cracks above a certain length never relax to a steady state, i.e., catastrophic failure. For strain hardening, $\alpha<0$, there is no localization of deformation. For intermediate values of slip weakening, 0 $<\alpha<1$, a complex population of faults emerges.

The model offers a new way to look at the evolution of populations of cracks. Three regimes are observed with increasing strain. At very low strain, the cracks are short, the crack population is dilute, there is very little interaction amongst the stress fields around them, and disorder dominates. The distributions here are exponential, with the scale of the exponential changing with increasing crack density. With additional extension, the cracks propagate in the direction normal to the direction of extension. Disorder and weakening compete, and the distribution of sizes of cracks is characterized by a power law. Only at the beginning do cracks grow solely by propagation of their tips, because they soon start to interact with other cracks. The stress field interactions shield unfavorably positioned cracks, which no longer accumulate slip or grow in length, and also inhibit nucleation of new cracks. As the population of cracks self-organizes, cracks begin to coalesce, shifting the main process of growth from propagation to coalescence. Their stress fields merge to form larger stress-free zones of trapped, dead cracks. The coalescence of the cracks as a result of the higher strain signifies a transition in the organization of the cracks to a regime in which the largest cracks have an exponential dis- tribution. As the cracks grow longer, shadow zones extend over a distance that scales with the depth of the brittle layer. The system reaches the maximum number of cracks allowed according to the stress interactions, i.e., saturation, characterized by evenly spaced system sized cracks. All three regimes are observed in natural systems, and in a physical model.

The model also suggests that, all other things being equal, faults that creep and faults that have earthquakes evolve in the same way. This is because the fault growth interactions occur with the accumulated strain, not strain increments. Therefore, we need not model earthquakes in order to study fault dynamics and fault interactions. Separating the two time scales of the problem is a valid approximation. Finally, the regime characterized by power laws occupies only a portion of the phase space of this system, although this is the portion most commonly observed for faults and earthquakes.

\section{ACKNOWLEDGMENTS}

We are grateful to $M$. Speigelman for a series of lengthy and useful discussions. We also thank P. Richards, B. Menke, M. Anders, P. Cowie, A. Gupta, and I. Manighetti for their help with geological observations and theory. This work was supported by the NSF through Grant Nos. EAR-94-17700, EAR-99-09287, and EAR-00-87645, by the USGS through Grant No. 1434-HQ-97-6R03074, by the SCEC through Grant No. 569934, and by the Center for Nonlinear Earth Systems, Columbia University.
[1] M. S. Hu and A. G. Evans, Acta Metall. 37, 917 (1989).

[2] C. H. Scholz and P. A. Cowie, Nature (London) 376, 837 (1990).

[3] C. H. Scholz, N. H. Dawers, J. Z. Yu, M. H. Anders, and P. A. Cowie, J. Geophys. Res. 98, 21951 (1993).

[4] C. H. Scholz, Bull. Seismol. Soc. Am. 87, 1074 (1997).

[5] C. H. Scholz, Bull. Seismol. Soc. Am. 88, 1325 (1998).

[6] P. Bak, C. Tang, and K. Wiesenfeld, Phys. Rev. A 38, 364 (1988).

[7] K. Chen, P. Bak, and S. P. Obukhov, Phys. Rev. A 43, 625 (1991).

[8] A. Sornette, P. Davy, and D. Sornette, Phys. Rev. Lett. 65, 2266 (1990).

[9] D. Sornette, P. Davy, and A. Sornette, J. Geophys. Rev. 95, 17353 (1990).

[10] P. A. Cowie, D. Sornette, and C. Vanneste, Geophys. J. Int. 122, 457 (1995).

[11] P. A. Cowie, C. H. Scholz, C. H. Edwards, and A. Malinverno, J. Geophys. Res., [Solid Earth] 98, 17911 (1993).
[12] H. Colina, L. de Arcangelis, and S. Roux, Phys. Rev. B 48, 3666 (1993).

[13] J. S. Langer, J. M. Carlson, C. R. Myers, and B. E. Shaw, Proc. Natl. Acad. Sci. U.S.A. 93, 3825 (1996).

[14] B. E. Shaw, J. Geophys. Res., [Solid Earth] 100, 18239 (1995).

[15] J. M. Vermilye and C. H. Scholz, J. Geophys. Res. 103, 12223 (1998).

[16] M. L. Falk and J. S. Langer, Phys. Rev. E 57, 7192 (1998).

[17] D. McKenzie, P. G. Ford, C. Johnson, B. Parsons, D. Sandwell, S. Saunders, and S. C. Solomon, J. Geophys. Res., [Solid Earth Planets] 97, 13533 (1992).

[18] S. E. Smreaker and S. C. Solomon, J. Geophys. Res., [Solid Earth Planets] 97, 16121 (1992).

[19] V. Ansan and P. Blondel, Planet. Space Sci. 44, 833 (1996).

[20] N. J. Price, Fault and Joint Development in Brittle and SemiBrittle Rock (Pergamon, New York, 1966).

[21] A. Gupta and C. H. Scholz, Geology 28, 1087 (2000).

[22] C. Spyropoulos, W. J. Griffith, C. H. Scholz, and B. E. Shaw, Geophys. Res. Lett. 26, 1081 (1999). 